\title{
Attribute reduction based on maximal rules in decision formal context
}

\author{
Leijun $\mathrm{Li}^{a, b}$, Jusheng $\mathrm{Mi}^{a *}, \mathrm{Bin} \mathrm{Xie}^{a}$ \\ ${ }^{a}$ College of Mathematics and Information Science, \\ Hebei Normal University, Shijiazhuang, 050016, P. R. China \\ ${ }^{b}$ Harbin Institute of Technology, Harbin, 150001, P. R. China \\ E-mail: lileijun1985@163.com,mijsh@263.net,happyxiebin@126.com
}

Received 11 June 2013

Accepted 24 May 2014

\begin{abstract}
One of the key issues in the theory of concept lattices is to extract the useful rules from the decision formal context. The maximal rules implicate the others, thus people are interested in them. This paper proposes two new kinds of attribute reduction in the decision formal context based on maximal rules. The reducts preserve all the condition extensions and the decision extensions related to the original maximal rules. The internal relationship between the original maximal rules and the maximal rules in the reduced decision formal context is derived. The reducts can make the maximal rules more concise and accurate. The mathematical property of the proposed attribute reduction is investigated and we construct the discernibility matrix and function to compute all the reducts. Finally, all the attributes are classified into three types based on the maximal rules. The characteristics of these types of attributes are also analyzed.
\end{abstract}

Keywords: concept lattices; maximal rule; condition reduct; decision reduct

\section{Introduction}

The theory of concept lattices (also called formal concept analysis), proposed by Wille in 1982, is a relatively new model to represent the formal concepts associated with a context ${ }^{30}$. It can unravel hierarchical concepts organized as a lattice from relational information systems. A formal context consists of a universe of discourse $U$, an attributes set $A$, and a binary relation $I$ from $U$ to $A$. Al1 the formal concepts are constructed from the formal context and form a concept lattice which reflects the relationship of generalization and the specialization among concepts. It has been studied from various perspectives, such as its mathematical property $4,8,13,17,18,25$ and the applications in knowledge discovery $3,5,6,26,33,35$.
In this paper, we investigate attribute reduction based on maximal rules in the decision formal context. Attribute reduction is performed in information systems by means of the notion of a reduct based on a specialization of the general notion of independence ${ }^{15}$. After eliminating the superfluous attributes, the formal context becomes simpler and the discovery of the initial knowledge hidden in the formal context becomes easier, thus it is a key problem in knowledge discovery. In recent years, many scholars have studied attribute reduction extensively from various perspectives and each of them aims at some basic requirements $14,16,19,27,32,36$. In 2005 , a method to attribute reduction was proposed in concept lattices via the discernibility matrix and Boolean function ${ }^{36}$. It can get all the reducts and each of them preserves all the original concepts and 
their hierarchy in the reduced context. Then inspired by rough set theory ${ }^{22}$, two kinds of attribute reduction methods and object reduction methods for concept lattices were developed, respectively ${ }^{14}$. After that, based on irreducible elements in the objec$\mathrm{t}$ and property oriented concept lattices, a new approach to attribute reduction was presented ${ }^{27}$. Recently, the relation among attribute reductions in formal, object-oriented and property-oriented concept lattices is studied and the equivalence among them has also been proved ${ }^{16}$. Besides, much attention has been paid to attribute reduction of the decision formal context $11,12,28,29,34$. A decision formal context consists of a universe of discourse $U$, a conditional attributes set $A$, a decision attribute set $C$, and binary relations $I$ from $U$ to $A$ and $J$ from $U$ to $C$. Based on the discernibility matrix and Boolean function, attribute reduction of the strongly consistent decision formal context and the weakly consistent decision formal context was discussed ${ }^{29}$. Then a knowledgereduction heuristic algorithm is developed to search for a minimal reduct of a consistent decision formal context and its time complexity is polynomial ${ }^{11}$. Recently, an order-preserving mapping is proposed to classify the decision formal contexts into consistent and inconsistent formal decision contexts. Then their corresponding knowledge reduction methods are given by constructing the suitable discernibility matrices and Boolean functions ${ }^{12}$.

On the other hand, rules extraction is an important problem and it has been studied extensively from different perspectives $1,2,9,10,20,21,23$. In the decision formal context, a rule is derived based on the condition formal concept (generated from the condition attribute set) and the decision formal concept (generated from the decision attribute set). Each formal concept is constituted with the extension and the intension. For a rule, there often exist some redundant attributes, thus the reduction based on rules extraction is necessary. As we know, the maximal rules implicate the other rules, thus the formal concepts related to the maximal rules are important. Attribute reduction based on maximal rules is proposed in this paper. The proposed reducts preserve all the condition extensions and decision extensions related to the original maximal rules. Furthermore, it is proven that all the maximal rules in the reduced decision formal are derived from these condition extensions and decision extensions. In other words, every original maximal rule corresponds to a maximal rule in the reduced decision formal context and they are derived based on the same condition extension and decision extension. However, the depiction of the maximal rule becomes more concise and accurate after eliminating the superfluous attributes. Using the similar idea of attribute reduction in the classical formal context ${ }^{36}$, the corresponding discernibility matrix is presented. All attributes are classified into three types based on the maximal rules. The characteristics of these types of attributes are then analyzed.

The remainder of the paper is organized as follows: in the next section, some basic concepts related to this paper are reviewed. In Section 3, two kind$\mathrm{s}$ of reduction based on maximal rules are proposed and their properties are investigated. In Section 4, we study attribute reduction based on maximal rules in the decision formal context. Some judgment theorems of a reduct are developed, and the discernibility matrix is constructed to compute all the reducts via Boolean approach. In Section 5, all the attributes are classified into three types based on maximal rules. The characteristics of these attributes are also studied. Finally, in Section 6, we conclude the paper with a summary and an outlook for further research.

\section{Preliminaries}

To facilitate our discussion, some basic notions and results related to this paper are reviewed in this section $7,29,31$.

A formal context is a triplet $(U, A, I)$, where $U$ is a nonempty finite set of objects, $A$ is a nonempty finite set of attributes, and $I \subseteq U \times A$ is a binary relation from $U$ to $A$. $(x, a) \in I$ means that $x$ has the attribute $a$.

Definition 1. Let $(U, A, I)$ be a formal context. For all $X \subseteq U$ and $B \subseteq A$, two operations are defined respectively

$$
\begin{aligned}
& X^{*}:=\{a \in A \mid \forall x \in X,(x, a) \in I\}, \\
& B^{*}:=\{x \in U \mid \forall a \in B,(x, a) \in I\} .
\end{aligned}
$$


Evidently, $X^{*}$ consists of exactly those attributes which the objects in $X$ have in common, and $B^{*}$ contains exactly those objects which share all attributes in $B$.

Definition 2. Let $(U, A, I)$ be a formal context. A pair $(X, B)$ with $X \subseteq U$ and $B \subseteq A$ is called a formal concept iff $X^{*}=B$ and $B^{*}=X$. Here $X$ and $B$ are called the extension and the intension of $(X, B)$, respectively.

Theorem 1. Let $(U, A, I)$ be a formal context. For all $X, X_{1}, X_{2} \subseteq U$ and $B, B_{1}, B_{2} \subseteq A$, the following statements hold:

(1) $X_{1} \subseteq X_{2} \Longrightarrow X_{1}^{*} \supseteq X_{2}^{*} ; B_{1} \subseteq B_{2} \Longrightarrow B_{1}^{*} \supseteq B_{2}^{*}$.

(2) $X \subseteq X^{* *}, B \subseteq B^{* *}$.

(3) $X^{*}=X^{* * *}, B^{*}=B^{* * *}$.

(4) $\left(X_{1} \cup X_{2}\right)^{*}=X_{1}^{*} \cap X_{2}^{*}$, $\left(B_{1} \cup B_{2}\right)^{*}=B_{1}^{*} \cap B_{2}^{*}$.

(5) $\left(X^{* *}, X^{*}\right)$ and $\left(B^{*}, B^{* *}\right)$ are both concepts.

Here $\left(X_{1}, B_{1}\right) \leqslant\left(X_{2}, B_{2}\right)$ if and only if $X_{1} \subseteq X_{2}$ (or equivalently $B_{2} \subseteq B_{1}$ ). The set of all the concepts in $(U, A, I)$ together with the partial order is denoted by $L(U, A, I)$. Furthermore, the set of all the extensions is defined as $L_{U}(U, A, I)=\{X \mid(X, B) \in$ $L(U, A, I)\}$. It can be seen that $L_{U}(U, A, I)=$ $\{X \mid(X, B) \in L(U, A, I)\}=\left\{X^{* *} \mid X \subseteq U\right\}$.

Definition 3. A five-tuple $(U, A, I, C, J)$ is called a decision formal context, if $(U, A, I)$ and $(U, C, J)$ are both the formal contexts, and $A$ is called a condition attribute set, $C$ is a decision attribute set.

Let $(U, A, I, C, J)$ be a decision formal contex$\mathrm{t}$, with each $B \subseteq A$, we associate a binary relation $I_{B}=I \cap(U \times B)$. Then $\left(U, B, I_{B}\right)$ is a sub-context of $(U, A, I)$. It is easy to see that $I_{A}=I, X^{*_{B}}=$ $X^{*} \cap B, X^{* B} \subseteq X^{*}$.

The following theorem follows immediately from the definition of $L_{U}(U, A, I)$.

Theorem 2. Let $(U, A, I)$ be a formal context. For all $B \subseteq A(B \neq \emptyset), L_{U}\left(U, B, I_{B}\right) \subseteq L_{U}(U, A, I)$.

Definition 4. Let $(X, K) \in L(U, A, I)$ and $(Y, H) \in$ $L(U, C, J)$. If $X \subseteq Y(Y \neq U, \emptyset)$, then " $K \rightarrow H$ " is called a rule. Furthermore, if there exist $\left(X^{\prime}, K^{\prime}\right) \in$ $L(U, A, I)$ and $\left(Y^{\prime}, H^{\prime}\right) \in L(U, C, J)$ satisfying $K \subseteq$ $K^{\prime}$ and $H \supseteq H^{\prime}$ (or equivalently $X^{\prime} \subseteq X$ and $Y^{\prime} \supseteq Y$ ), then it means that " $K \rightarrow H$ " implicates " $K^{\prime} \rightarrow H^{\prime}$ ", and the rule " $K$ ' $\rightarrow H^{\prime}$ " is redundant. If there is no rule " $K^{\prime} \rightarrow H^{\prime}$ ", satisfying $K^{\prime} \subseteq K, H \subseteq H^{\prime}$, then the rule " $K \rightarrow H$ " is called a maximal rule.

It should be noted that the rule proposed above is in fact a decision implication ${ }^{24}$. However, a decision implication may not be a rule because it$\mathrm{s}$ premise or conclusion may not be the intension of any formal concept. Besides, the premise or the conclusion of the implication rules given in ${ }^{7}$ may also not be the intension of any formal concept. Thus, compared with the decision implication ${ }^{24}$ and implication rules ${ }^{7}$, the rule proposed above has more semantic explanation than a decision implication.

\section{Attribute reduction based on maximal rules}

In this section, we introduce the definition of attribute reduction based on maximal rules in the decision formal context, and then present some properties related to the definition.

For any $X_{i} \in L_{U}(U, A, I)$, denote by $S_{C}\left(X_{i}\right)=$ $\left\{Y_{j} \mid X_{i} \subseteq Y_{j} \in L_{U}(U, C, J)\right.$ and for any $Y_{j}^{\prime} \in$ $L_{U}(U, C, J)$, if $X_{i} \subseteq Y_{j}^{\prime}$, then $\left.Y_{j}^{\prime} \not \subset Y_{j}\right\}$.

Clearly, for any $Y_{j} \in S_{C}\left(X_{i}\right), Y_{j}$ is the minimal extension which contains $X_{i}$ in $L_{U}(U, C, J)$ and denote by

$$
\Phi_{C}=\bigcup_{X_{i} \in L_{U}(U, A, I)} S_{C}\left(X_{i}\right) .
$$

For any $Y_{j} \in \Phi_{C}, \quad T_{A}\left(Y_{j}\right)=\left\{X_{i} \mid Y_{j} \supseteq X_{i} \in\right.$ $L_{U}(U, A, I)$ and for any $X_{i}^{\prime} \in L_{U}(U, A, I)$, if $X_{i}^{\prime} \subseteq$ $Y_{j}$, then $\left.X_{i}^{\prime} \not \supset X_{i}\right\}$ and denote by

$$
\Psi_{A}=\bigcup_{Y_{j} \in \Phi_{C}} T_{A}\left(Y_{j}\right)
$$

Evidently, for any $X_{i} \in T_{A}\left(Y_{j}\right), X_{i}$ is the maximal extension which is contained in $Y_{j}$ in $L_{U}(U, A, I)$.

From the definition of $\Phi_{C}$ and $T_{A}(Y)$, the following theorem can be obtained:

Theorem 3. Let $(U, A, I, C, J)$ be a decision formal context. For any $(X, K) \in L(U, A, I)$ and $(Y, H) \in$ $L(U, C, J)$, " $K \rightarrow H$ " is a maximal rule if and only if $Y \in S_{C}(X)(Y \neq U, \emptyset)$ and $X \in T_{A}(Y)$.

Definition 5. Let $(U, A, I, C, J)$ be a decision formal context. $D \subseteq C$ is called a decision consistent set 
if for all $X_{i} \in L_{U}(U, A, I), S_{D}\left(X_{i}\right)=S_{C}\left(X_{i}\right)$. Furthermore, if for any $d \in D$, there exists $X_{i} \in L_{U}(U, A, I)$ such that $S_{D \backslash\{d\}}\left(X_{i}\right) \neq S_{C}\left(X_{i}\right)$, then $D$ is called a decision reduct of $(U, A, I, C, J)$. The intersection of all the decision reducts are called the decision core of $(U, A, I, C, J)$.

A decision reduct $D$ of $(U, A, I, C, J)$ is a minimal attribute subset satisfying for all $X_{i} \in L_{U}(U, A, I)$, $S_{D}\left(X_{i}\right)=S_{C}\left(X_{i}\right)$.

Definition 6. Let $(U, A, I, C, J)$ be a decision formal context, $E \subseteq A$ is called a condition consisten$\mathrm{t}$ set if for all $Y_{j} \in \Phi_{C}, T_{E}\left(Y_{j}\right)=T_{A}\left(Y_{j}\right)$. Furthermore, if for any $e \in E$ there exists $Y_{j} \in \Phi_{C}$ such that $T_{E \backslash\{e\}}\left(Y_{j}\right) \neq T_{A}\left(Y_{j}\right)$, then $E$ is called a condition reduct of $(U, A, I, C, J)$. The intersection of all the condition reducts are called the condition core of $(U, A, I, C, J)$.

A condition reduct $E$ of $(U, A, I, C, J)$ is a minimal attribute subset satisfying for all $Y_{j} \in \Phi_{C}$, $T_{E}\left(Y_{j}\right)=T_{A}\left(Y_{j}\right)$.

It follows from the Definitions 5 and 6 that there are at least one condition reduct and one decision reduct in the decision formal context.

We can see that the reducts preserve all the condition extensions and the decision extensions related to the original maximal rules. And further, the following theorem is given:

Theorem 4. Let $(U, A, I, C, J)$ be a decision formal context, $D \subseteq C$ is a decision consistent set and $E \subseteq A$ is a condition consistent set. Then for all $(X, K) \in L\left(U, E, I_{E}\right),(Y, H) \in L\left(U, D, J_{D}\right), " K \rightarrow H$ " is a maximal rule in $\left(U, E, I_{E}, D, J_{D}\right)$ if and only if $Y \in S_{C}(X), X \in T_{A}(Y)$.

Proof. $\Rightarrow)$ " $K \rightarrow H$ " is a maximal rule in $\left(U, E, I_{E}, D, J_{D}\right)$, thus $Y \in S_{D}(X)$ and $X \in T_{E}(Y)$. From Theorem 2 and the definition of decision consistent set, $X \in L_{U}\left(U, E, I_{E}\right) \subseteq L_{U}(U, A, I), S_{D}(X)=$ $S_{C}(X)$, thus $Y \in S_{C}(X)$. From the definition of the condition consistent set and $Y \in S_{C}(X)$, we have that $X \in T_{E}(Y)=T_{A}(Y)$.

$\Leftarrow)$ From the definition of $S_{C}(X)$ and $T_{A}(Y)$, there is no $X^{\prime} \in L_{U}(U, A, I)$ and $Y^{\prime} \in L_{U}(U, C, J)$ satisfying $X \subset X^{\prime} \subseteq Y$ and $X \subset Y^{\prime} \subseteq Y$. By Theorem 2, $L_{U}\left(U, E, I_{E}\right) \subseteq L_{U}(U, A, I)$ and $L_{U}\left(U, D, J_{D}\right) \subseteq$ $L_{U}(U, C, J)$ hold. Thus, there is no $X^{\prime} \in L_{U}\left(U, E, I_{E}\right)$ and $Y^{\prime} \in L_{U}\left(U, D, J_{D}\right)$ satisfying $X \subset X^{\prime} \subseteq Y$ and $X \subset Y^{\prime} \subseteq Y$. Therefore, " $K \rightarrow H$ " is a maximal rule of $\left(U, E, I_{E}, D, J_{D}\right)$.

From Theorem 3, we know that, if $Y \in \Phi_{C}, X \in$ $T_{A}(Y)$, then " $X^{* A} \rightarrow Y^{* C \text { " }}$ is a maximal rule in $(U, A, I, C, J)$. Thus, it can be seen that " $X^{* A} \rightarrow Y^{* C}$ " and " $K \rightarrow H$ " are derived based on the same condition extensions and decision extensions. However,

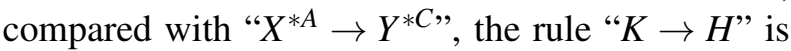
more concise and accurate.

Furthermore, if $(X, K) \in L\left(U, E, I_{E}\right),(Y, H) \in$ $L\left(U, D, J_{D}\right)$, then $K=X^{* A} \cap E, H=Y^{* C} \cap D$. We can see that the maximal rule " $K \rightarrow H$ " in the reduced decision formal context $\left(U, E, I_{E}, D, J_{D}\right)$ can be obtained based on the original maximal rule " $X^{* A} \rightarrow Y^{* C "}$, the condition consistent set $E$ and the decision consistent set $D$. What is more, every original maximal rule corresponds to a maximal rule in the reduced decision formal context. For example, if " $X^{* A} \rightarrow Y^{* C}$ " is a maximal rule in $(U, A, I, C, J)$, then from Theorem 3, $Y \in S_{C}(X), X \in T_{A}(Y) . E \subseteq A$ and $D \subseteq C$ are the condition consistent set and the decision consistent set, thus $X \in T_{A}(Y)=T_{E}(Y)$ and $Y \in$ $S_{C}(X)=S_{D}(X)$. Therefore, " $X^{* A} \cap E \rightarrow Y^{* C} \cap D$ " is a maximal rule in $\left(U, E, I_{E}, D, J_{D}\right)$.

In what follows, we only consider the reduction method and attribute characteristics of decision attributes in detail. As to condition attributes, we only give its discernibility matrix and the other related problems can be discussed as the decision attributes.

\section{Approaches to attribute reduction based on maximal rules}

In this section, inspired by the approaches to attribute reduction in classical concept lattices, we construct the discernibility matrix in the decision formal context based on maximal rules, and then obtain all the reducts via Boolean approach.

Definition 7. Let $(U, A, I, C, J)$ be a decision formal context. For all $\left(Y_{i}, B_{i}\right),\left(Y_{j}, B_{j}\right) \in L(U, C, J)$,

$$
\begin{aligned}
& D\left(\left(Y_{i}, B_{i}\right),\left(Y_{j}, B_{j}\right)\right)= \\
& \begin{cases}B_{i} \triangle B_{j}, & \text { if } Y_{i} \in \Phi_{C}, Y_{i} \subset Y_{j} \text { or } Y_{j} \in \Phi_{C}, Y_{j} \subset Y_{i} \\
\emptyset, & \text { else }\end{cases}
\end{aligned}
$$


is called the discriminable attribute set between $\left(Y_{i}, B_{i}\right)$ and $\left(Y_{j}, B_{j}\right)$. Here $B_{i} \triangle B_{j}=B_{i} \cup B_{j}-B_{i} \cap B_{j}$ and $\Lambda_{F C}=\left(D\left(\left(Y_{i}, B_{i}\right),\left(Y_{j}, B_{j}\right)\right) \mid\left(Y_{i}, B_{i}\right),\left(Y_{j}, B_{j}\right) \in\right.$ $L(U, C, J))$ is called the discernibility matrix of the decision attributes. Denote by

$D_{0}=\left\{D\left(\left(Y_{i}, B_{i}\right),\left(Y_{j}, B_{j}\right)\right) \mid D\left(\left(Y_{i}, B_{i}\right),\left(Y_{j}, B_{j}\right)\right) \neq \emptyset\right\}$

In what follows, some judgment theorems of the decision consistent sets are proposed.

Theorem 5. Let $(U, A, I, C, J)$ be a decision formal context. For all $D \subseteq C(D \neq \emptyset)$, the following statements are equivalent.

(1) $D$ is a decision consistent set.

(2) $\Phi_{C} \subseteq L_{U}\left(U, D, J_{D}\right)$.

(3) For $\left(Y_{i}, B_{i}\right),\left(Y_{j}, B_{j}\right) \in L(U, C, J)$, if $Y_{i} \in$ $\Phi_{C}, Y_{i} \subset Y_{j}\left(\right.$ or $\left.Y_{j} \in \Phi_{C}, Y_{j} \subset Y_{i}\right)$, then $B_{i} \cap D \neq$ $B_{j} \cap D$.

(4) $D \cap D\left(\left(Y_{i}, B_{i}\right),\left(Y_{j}, B_{j}\right)\right) \neq \emptyset\left(\forall D\left(\left(Y_{i}, B_{i}\right),\left(Y_{j}\right.\right.\right.$, $\left.\left.\left.B_{j}\right)\right) \neq \emptyset\right)$.

(5) For all $B \subseteq C$, if $B \cap D=\emptyset$, then $B \notin D_{0}$.

\section{Proof.}

$(1) \Leftrightarrow(2)$ It follows immediately from the definition of decision consistent set.

$(2) \Rightarrow(3)$ For $\left(Y_{i}, B_{i}\right),\left(Y_{j}, B_{j}\right) \in L(U, C, J)$, without loss of generality, we assume that $Y_{i} \in \Phi_{C}$ and $Y_{i} \subset Y_{j}$. Since $\Phi_{C} \subseteq L_{U}\left(U, D, J_{D}\right)$, then there exists $\left(Y_{i}, B_{i}^{\prime}\right) \in L\left(U, D, J_{D}\right)$ with $B_{i}^{\prime}=B_{i} \cap D$. Assume that $B_{i} \cap D=B_{j} \cap D$, then $Y_{j} \subseteq\left(B_{j} \cap D\right)^{*}=\left(B_{i} \cap D\right)^{*}=$ $Y_{i} \subset Y_{j}$, which contradicts to the fact that $Y_{j}=Y_{j}$. Therefore, $B_{i} \cap D \neq B_{j} \cap D$.

(3) $\Rightarrow(2)$ For $(Y, B) \in \Phi_{C}$, we need to prove $(Y, B \cap D) \in L\left(U, D, J_{D}\right)$.

On the one hand, it is easy to see that $Y^{* D}=$ $B \cap D$.

On the other hand, assume that $(B \cap D)^{*} \neq Y$, then $(B \cap D)^{*} \supset Y$. Since $\left((B \cap D)^{*},(B \cap D)^{* *}\right)$ $\in L(U, C, J),(B \cap D)^{* *} \cap D \neq B \cap D$ holds by (3). Since $B \cap D \subseteq B \Rightarrow(B \cap D)^{*} \supseteq B^{*}=Y \Rightarrow(B \cap D)^{* *} \subseteq$ $Y^{*}=B \Rightarrow(B \cap D)^{* *} \cap D \subseteq B \cap D$, and $B \cap D \subseteq$ $(B \cap D)^{* *} \Rightarrow B \cap D \subseteq(B \cap D)^{* *} \cap D$, we conclude that $B \cap D=(B \cap D)^{* *} \cap D$, which contradicts to the fact that $B \cap D \neq(B \cap D)^{* *} \cap D$. Therefore, $(B \cap D)^{*}=Y$.

(3) $\Rightarrow(4)$ For $\left(Y_{i}, B_{i}\right),\left(Y_{j}, B_{j}\right) \in L(U, C, J)$, if $D\left(\left(Y_{i}, B_{i}\right),\left(Y_{j}, B_{j}\right)\right) \neq \emptyset$, which implies that $Y_{i} \in$ $\Phi_{C}, Y_{i} \subset Y_{j}$ or $Y_{j} \in \Phi_{C}, Y_{j} \subset Y_{i}$, thus $B_{i} \cap D \neq B_{j} \cap D$ holds by (3). And further, $B_{i} \cap D-B_{j} \cap D=$ $D \cap B_{i} \cap \overline{B_{j}} \neq \emptyset$, or $B_{j} \cap D-B_{i} \cap D=D \cap B_{j} \cap \overline{B_{i}} \neq$ $\emptyset$. Therefore, we have that

$$
\begin{aligned}
D \cap D\left(\left(Y_{i}, B_{i}\right),\left(Y_{j}, B_{j}\right)\right) & =D \cap\left(B_{i} \cup B_{j}-B_{i} \cap B_{j}\right) \\
& \left.=D \cap\left(B_{i} \cup B_{j}\right) \cap \overline{B_{i}} \cup \overline{B_{j}}\right) \\
& =\left(D \cap B_{i} \cap \overline{B_{j}}\right) \cup\left(D \cap B_{i} \cap \overline{B_{j}}\right) \\
& \neq \emptyset .
\end{aligned}
$$

(4) $\Rightarrow$ (3) For $\left(Y_{i}, B_{i}\right),\left(Y_{j}, B_{j}\right) \in L(U, C, J)$, if $Y_{i} \in \Phi_{C}, Y_{i} \subset Y_{j}$ or $Y_{j} \in \Phi_{C}, Y_{j} \subset Y_{i}$, then $D\left(\left(Y_{i}, B_{i}\right),\left(Y_{j}, B_{j}\right)\right) \neq \emptyset$ holds by Definition 7 , thus $D \bigcap D\left(\left(Y_{i}, B_{i}\right),\left(Y_{j}, B_{j}\right)\right) \neq \emptyset$. Therefore, there exists $d \in D \cap D\left(\left(Y_{i}, B_{i}\right),\left(Y_{j}, B_{j}\right)\right)$, which implies $d \in B_{i}$ or $d \in B_{j}$, and $d \notin B_{i} \cap B_{j}$. If $d \in B_{i}$ and $d \notin B_{j}$, then $d \in D \cap B_{i}$ and $d \notin D \cap B_{j}$; If $d \in B_{j}$ and $d \notin B_{i}$, then $d \in D \cap B_{j}$ and $d \notin D \cap B_{i}$. Therefore, $B_{i} \cap D \neq B_{j} \cap D$.

(4) $\Leftrightarrow(5)$ It follows immediately from the definition of $D_{0}$.

From Theorem 5, we know that the decision reduct of the decision formal context $(U, A, I, C, J)$ is a minimal subset $D$ of $C$ such that $D \cap$ $D\left(\left(Y_{i}, B_{i}\right),\left(Y_{j}, B_{j}\right)\right) \neq \emptyset\left(\forall D\left(\left(Y_{i}, B_{i}\right),\left(Y_{j}, B_{j}\right)\right) \neq \emptyset\right)$.

Furthermore, the discernibility function is given based on its discernibility matrix.

$$
f\left(D_{0}\right)=\bigwedge_{H \in D_{0}}\left(\bigvee_{h \in H} h\right)
$$

By the absorption law and the distributive law, $f\left(D_{0}\right)$ can be translated to the minimal disjunctive normal form. From Theorem 5 and the definition of the minimal disjunctive normal form, it follows immediately that the components of conjunctive normal forms are all the decision reducts of the decision formal context $(U, A, I, C, J)$.

The discernibility matrix of the condition attributes is given as follows and its reducts can be computed as the procedure of the decision reducts.

Definition 8. Let $(U, A, I, C, J)$ be a decision formal context. For all $\left(X_{i}, K_{i}\right),\left(X_{j}, K_{j}\right) \in L(U, A, I)$,

$$
\begin{aligned}
& D\left(\left(X_{i}, K_{i}\right),\left(X_{j}, K_{j}\right)\right)= \\
& \begin{cases}K_{i} \triangle K_{j}, & \text { if } X_{i} \in \Psi_{A}, X_{i} \subset X_{j} \text { or } X_{j} \in \Psi_{A}, X_{j} \subset X_{i} \\
\emptyset, & \text { else. }\end{cases}
\end{aligned}
$$

is called the discriminable attribute set between $\left(X_{i}, K_{i}\right)$ and $\left(X_{j}, K_{j}\right)$. Here $K_{i} \triangle K_{j}=K_{i} \cup K_{j}-K_{i} \cap$ 
$K_{j}$ and $\Lambda_{F C}=\left(D\left(\left(X_{i}, K_{i}\right),\left(X_{j}, K_{j}\right)\right) \mid\left(X_{i}, K_{i}\right),\left(X_{j}, K_{j}\right)\right.$ $\in L(U, A, I))$ is called the discernibility matrix of the condition attributes.

The following example illustrates our idea of attribute reduction based on maximal rules in further.

Example 1. A decision formal context $S=$ $(U, A, I, C, J)$ is shown in Table 1 , where the condition attributes set $A=\{a, b, c, d, e, f\}$ and the decision attributes set $C=\{g, h, k, l, m, n\}$ ).

Table 1. A decision formal context $S=(U, A, I, C, J)$

\begin{tabular}{ccccccccccccc}
\hline & $a$ & $b$ & $c$ & $d$ & $e$ & $f$ & $g$ & $h$ & $k$ & $l$ & $m$ & $n$ \\
\hline 1 & 1 & 1 & 1 & 0 & 0 & 1 & 1 & 1 & 1 & 0 & 1 & 1 \\
2 & 1 & 0 & 1 & 1 & 1 & 1 & 0 & 0 & 1 & 0 & 0 & 0 \\
3 & 0 & 0 & 0 & 1 & 0 & 0 & 0 & 1 & 0 & 1 & 0 & 1 \\
4 & 0 & 1 & 1 & 0 & 1 & 1 & 1 & 0 & 0 & 0 & 1 & 0 \\
5 & 1 & 0 & 1 & 1 & 0 & 1 & 0 & 0 & 0 & 0 & 1 & 1 \\
6 & 0 & 0 & 0 & 0 & 0 & 1 & 0 & 1 & 0 & 1 & 0 & 1 \\
\hline
\end{tabular}

In what follows, the condition concept lattice (Figure 1) and the decision concept lattice (Figure 2) are presented .

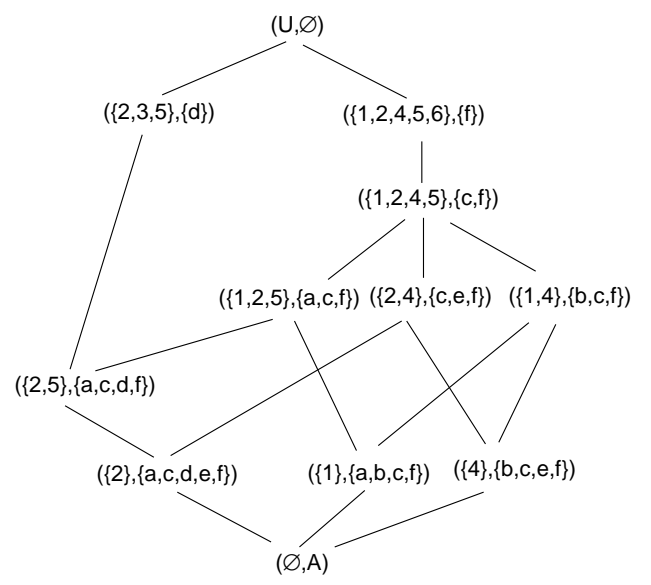

Fig.1. condition concept lattice

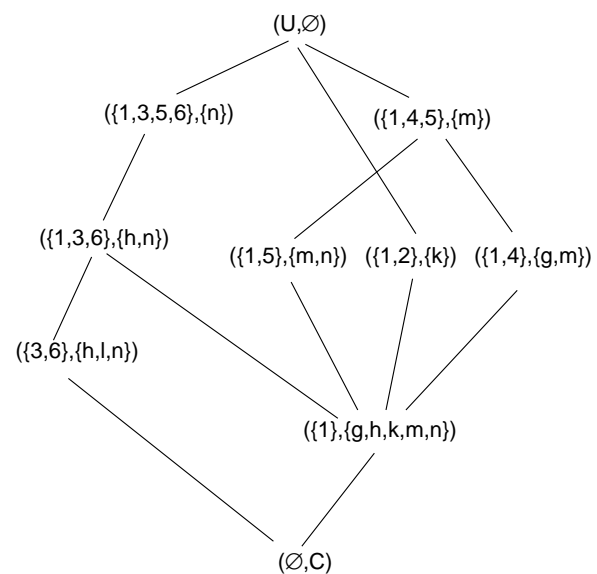

Fig.2. decision concept lattice

Clearly:

$L_{U}(U, A, I)=\left\{X_{1}=U, X_{2}=\{1,2,4,5,6\}, X_{3}=\right.$ $\{2,3,5\}, X_{4}=\{1,2,4,5\}, X_{5}=\{1,2,5\}, X_{6}=$ $\{2,5\}, X_{7}=\{2,4\}, X_{8}=\{1,4\}, X_{9}=\{4\}, X_{10}=$ $\left.\{2\}, X_{11}=\{1\}, X_{12}=\emptyset\right\}$;

$L_{U}(U, C, J)=\left\{Y_{1}=U, Y_{2}=\{1,3,5,6\}, Y_{3}=\right.$ $\{1,4,5\}, Y_{4}=\{1,3,6\}, Y_{5}=\{1,5\}, Y_{6}=\{1,2\}, Y_{7}=$ $\left.\{1,4\}, Y_{8}=\{3,6\}, Y_{9}=\{1\}, Y_{10}=\emptyset\right\}$.

Thus $S_{C}\left(X_{1}\right)=\left\{Y_{1}\right\}, S_{C}\left(X_{2}\right)=\left\{Y_{1}\right\}, S_{C}\left(X_{3}\right)=$ $\left\{Y_{1}\right\}, S_{C}\left(X_{4}\right)=\left\{Y_{1}\right\}, S_{C}\left(X_{5}\right)=\left\{Y_{1}\right\}, S_{C}\left(X_{6}\right)=$ $\left\{Y_{1}\right\}, S_{C}\left(X_{7}\right)=\left\{Y_{1}\right\}, S_{C}\left(X_{8}\right)=\left\{Y_{7}\right\}, S_{C}\left(X_{9}\right)=$ $\left\{Y_{7}\right\}, S_{C}\left(X_{10}\right)=\left\{Y_{6}\right\}, S_{C}\left(X_{11}\right)=\left\{Y_{9}\right\}, S_{C}\left(X_{12}\right)=$ $\left\{Y_{10}\right\}$.

Consequently, $\Phi_{C}=\left\{Y_{1}, Y_{6}, Y_{7}, Y_{9}, Y_{10}\right\}$.

Furthermore, $T_{A}\left(Y_{1}\right)=\left\{X_{1}\right\}, T_{A}\left(Y_{6}\right)=\left\{X_{10}\right\}, T_{A}($ $\left.Y_{7}\right)=\left\{X_{8}\right\}, T_{A}\left(Y_{9}\right)=\left\{X_{11}\right\}, T_{A}\left(Y_{10}\right)=\left\{X_{12}\right\}$.

Therefore, $\Psi_{A}=\left\{X_{1}, X_{8}, X_{10}, X_{11}, X_{12}\right\}$.

Then the maximal rules in the decision formal context $S=(U, A, I, C, J)$ can be given as follows:

(1) if $b \wedge c \wedge f$, then $g \wedge m$;

(2) if $a \wedge c \wedge d \wedge e \wedge f$, then $k$;

(3) if $a \wedge b \wedge c \wedge f$, then $g \wedge h \wedge k \wedge m \wedge n$.

Based on $\Psi_{A}$ and $\Phi_{C}$, the discernibility matrixs of the condition attributes and the decision attributes are presented as Tables 2 and 3, respectively.

Then the discernibility function of the condition attributes is 
Table 2: Discernibility matrix of condition attributes

\begin{tabular}{lllllllllllll}
\hline & $X_{1}$ & $X_{2}$ & $X_{3}$ & $X_{4}$ & $X_{5}$ & $X_{6}$ & $X_{7}$ & $X_{8}$ & $X_{9}$ & $X_{10}$ & $X_{11}$ & $X_{12}$ \\
\hline$X_{1}$ & $\emptyset$ & & & & & & & & & & & \\
$X_{2}$ & $\emptyset$ & $\emptyset$ & & & & & & & & & \\
$X_{3}$ & $\emptyset$ & $\emptyset$ & $\emptyset$ & & & & & & & & \\
$X_{4}$ & $\emptyset$ & $\emptyset$ & $\emptyset$ & $\emptyset$ & & & & & & & \\
$X_{5}$ & $\emptyset$ & $\emptyset$ & $\emptyset$ & $\emptyset$ & $\emptyset$ & & & & & & \\
$X_{6}$ & $\emptyset$ & $\emptyset$ & $\emptyset$ & $\emptyset$ & $\emptyset$ & $\emptyset$ & & & & & & \\
$X_{7}$ & $\emptyset$ & $\emptyset$ & $\emptyset$ & $\emptyset$ & $\emptyset$ & $\emptyset$ & $\emptyset$ & & & & & \\
$X_{8}$ & $\{$ bcf $\}$ & $\{$ bc $\}$ & $\emptyset$ & $\{b\}$ & $\emptyset$ & $\emptyset$ & $\emptyset$ & $\emptyset$ & & & & \\
$X_{9}$ & $\emptyset$ & $\emptyset$ & $\emptyset$ & $\emptyset$ & $\emptyset$ & $\emptyset$ & $\emptyset$ & $\emptyset$ & $\emptyset$ & & & \\
$X_{10}$ & $\{$ acdef $\}$ & $\{$ acde $\}$ & $\{$ acef $\}$ & $\{$ ade $\}$ & $\{$ de $\}$ & $\{$ e $\}$ & $\{$ ad $\}$ & $\emptyset$ & $\emptyset$ & $\emptyset$ & & \\
$X_{11}$ & $\{$ abcf $\}$ & $\{$ abc $\}$ & $\emptyset$ & $\{$ ab $\}$ & $\{$ b $\}$ & $\emptyset$ & $\emptyset$ & $\{$ a $\}$ & $\emptyset$ & $\emptyset$ & $\emptyset$ & \\
$X_{12}$ & A & $\{$ abcde $\}$ & $\{$ abcef $\}$ & $\{$ abde $\}$ & $\{$ bde $\}$ & $\{$ be $\}$ & $\{$ abd $\}$ & $\{$ ade $\}$ & $\{$ ad $\}$ & $\{$ b $\}$ & $\{$ de $\}$ & $\emptyset$ \\
\hline
\end{tabular}

Table 3: Discernibility matrix of decision attributes

\begin{tabular}{lllllllllll}
\hline & $Y_{1}$ & $Y_{2}$ & $Y_{3}$ & $Y_{4}$ & $Y_{5}$ & $Y_{6}$ & $Y_{7}$ & $Y_{8}$ & $Y_{9}$ & $Y_{10}$ \\
\hline$Y_{1}$ & $\emptyset$ & & & & & & & & & \\
$Y_{2}$ & $\emptyset$ & $\emptyset$ & & & & & & & \\
$Y_{3}$ & $\emptyset$ & $\emptyset$ & $\emptyset$ & & & & & & \\
$Y_{4}$ & $\emptyset$ & $\emptyset$ & $\emptyset$ & $\emptyset$ & & & & & \\
$Y_{5}$ & $\emptyset$ & $\emptyset$ & $\emptyset$ & $\emptyset$ & $\emptyset$ & & & & \\
$Y_{6}$ & $\{\mathrm{k}\}$ & $\emptyset$ & $\emptyset$ & $\emptyset$ & $\emptyset$ & $\emptyset$ & & & \\
$Y_{7}$ & $\{\mathrm{gm}\}$ & $\emptyset$ & $\{\mathrm{g}\}$ & $\emptyset$ & $\emptyset$ & $\emptyset$ & $\emptyset$ & & \\
$Y_{8}$ & $\emptyset$ & $\emptyset$ & $\emptyset$ & $\emptyset$ & $\emptyset$ & $\emptyset$ & $\emptyset$ & $\emptyset$ & & \\
$Y_{9}$ & $\{$ ghkmn $\}$ & $\{$ ghkm $\}$ & $\{$ ghkn $\}$ & $\{$ gkm $\}$ & $\{$ ghk $\}$ & $\{$ ghmn $\}$ & $\{$ hkn $\}$ & $\emptyset$ & $\emptyset$ & \\
$Y_{10}$ & $C$ & $\{$ ghklm $\}$ & $\{$ ghkln $\}$ & $\{$ gklm $\}$ & $\{$ ghkl\} & $\{$ ghlmn $\}$ & $\{$ hkln $\}$ & $\{$ gkm $\}$ & $\{1\}$ & $\emptyset$ \\
\hline
\end{tabular}

$$
\begin{aligned}
& \triangle_{1}=(b \vee c \vee f) \wedge(b \vee c) \wedge(b) \wedge(a \vee c \vee d \vee e \\
&f) \vee \wedge(a \vee c \vee d \vee e) \wedge(a \vee c \vee e \vee f) \wedge(a \\
&\vee d \vee e) \wedge(d \vee e) \wedge(e) \wedge(a \vee d) \wedge(a \vee b \vee \\
&c \vee f) \wedge(a \vee b \vee c) \wedge(a \vee b) \wedge(a) \wedge(a \vee b \\
&\vee c \vee d \vee e \vee f) \wedge(a \vee b \vee c \vee d \vee e) \wedge(a \vee \\
&b \vee d \vee e) \wedge(b \vee d \vee e) \wedge(b \vee e) \wedge(a \vee b \vee \\
&d) \wedge(a \vee b \vee c \vee e \vee f) \\
&= a \wedge b \wedge e .
\end{aligned}
$$

Therefore, $E=\{a, b, e\}$ is the unique condition reduct of the decision formal context $(U, A, I, C, J)$.

The discernibility function of the decision at- tributes is

$$
\begin{aligned}
\triangle_{2}= & (k) \wedge(g \vee m) \wedge(g) \wedge(g \vee h \vee k \vee m \vee n) \wedge \\
& (g \vee h \vee k \vee m) \wedge(g \vee h \vee k \vee n) \wedge(g \vee k \vee \\
& m) \wedge(g \vee h \vee k) \wedge(g \vee h \vee m \vee n) \wedge(h \vee k \\
& \vee n) \wedge(g \vee h \vee k \vee l \vee m \vee n) \wedge(g \vee h \vee k \\
& \vee l \vee m) \wedge(g \vee h \vee k \vee l \vee n) \wedge(g \vee k \vee l \vee \\
& m) \wedge(g \vee h \vee k \vee l) \wedge(g \vee h \vee l \vee m \vee n) \wedge \\
& (h \vee k \vee l \vee n) \wedge(l) \\
= & g \wedge k \wedge l .
\end{aligned}
$$

Therefore, $D=\{g, k, l\}$ is the unique decision reduct of the decision formal context $(U, A, I, C, J)$.

It is easy to see that $\Phi_{C} \subseteq L\left(U, D, J_{D}\right)$ and $\Psi_{A} \subseteq$ $L\left(U, E, I_{E}\right)$ in $\left(U, E, I_{E}, D, J_{D}\right)$.

The maximal rules in the reduced decision formal context $\left(U, E, I_{E}, D, J_{D}\right)$ can be obtained based on the original maximal rules, the condition reduct and the decision reduct. They are given as follows:

$\left(1^{\prime}\right)$ if $b$, then $g$; 
$\left(2^{\prime}\right)$ if $a \wedge e$, then $k$;

$\left(3^{\prime}\right)$ if $a \wedge b$, then $g \wedge k$.

These maximal rules are derived from the condition extensions and the decision extensions related to the original maximal rules. However, it can be seen that they are more concise and accurate.

\section{Attribute characteristics based on maximal rules}

Based on maximal rules, the significance of the decision attributes in a given decision formal contex$\mathrm{t}$ may be different and they play different roles in the decision reduct. These decision attributes can be classified into three types: the absolutely necessary decision attributes, the relatively necessary decision attributes, and the absolutely unnecessary decision attributes. In this section, we provide the characteristics of these types of attributes.

Definition 9. Let $S=(U, A, I, C, J)$ be a decision formal context and $D_{i} \subseteq C$, with $i \in \Upsilon$, are the decision reducts of $S$, then the attributes in $C$ are classified into three types:

(1) Absolutely necessary decision attribute set: $L=\bigcap_{i \in \Upsilon} D_{i}$.

(2) Relatively necessary decision attribute set: $M=\bigcup_{i \in \Upsilon} D_{i}-\bigcap_{i \in \Upsilon} D_{i}$.

(3) Absolutely unnecessary decision attribute set: $N=C-\bigcup_{i \in \Upsilon} D_{i}$.

It should be noted that the method of classifying the attributes has been proposed in ${ }^{36}$ and it is generalized to classify the attributes based on the maximal rules in this paper.

Denote by $\Phi_{C}=\{B \mid(Y, B) \in L(U, C, J)$ and $Y \in$ $\left.\Phi_{U}\right\}$, we get the following theorem.

Theorem 6. Let $S=(U, A, I, C, J)$ be a decision formal context. $D \subseteq C$ is a decision consistent set of $S$ if and only if for all $B \in \Phi_{C}, B^{*}=\left(B^{* *} \cap D\right)^{*}$ holds.

Proof. $\Rightarrow$ ) If $D$ is a decision consistent set, then $\Phi_{U} \subseteq L_{U}\left(U, D, J_{D}\right)$. Since for all $B \in \Phi_{C}$ we have $B^{*} \in \Phi_{U}$, then $B^{*} \in L_{U}\left(U, D, J_{D}\right)$, which means that $\left(B^{*}, B^{* *} \cap D\right) \in L\left(U, D, J_{D}\right)$, thus, $B^{*}=\left(B^{* *} \cap D\right)^{*}$.

$\Leftarrow)$ For all $Y \in \Phi_{U}$, we have $B \in \Phi_{C}$ such that $B^{*}=Y$. On the other hand, $B^{*}=\left(B^{* *} \cap D\right)^{*} \in$
$L_{U}\left(U, D, J_{D}\right)$, thus $\Phi_{U} \subseteq L_{U}\left(U, D, J_{D}\right)$. Therefore, $D$ is a decision consistent set.

Theorem 7. Let $S=(U, A, I, C, J)$ be a decision formal context, $d \in C$. Then

(1) $d$ is an absolutely necessary decision attribute iff there exists $B \in \Phi_{C}$ satisfying ( $B^{* *}-$ $\{d\})^{*} \neq B^{*}$.

(2) $d$ is a relatively necessary decision attribute iff for all $B \in \Phi_{C}$, satisfying $\left(B^{* *}-\{d\}\right)^{*}=B^{*}$ and there exists $D_{i_{0}}$, with $i_{0} \in \Upsilon$, and $K \in \Phi_{C}$ satisfying $\left(K^{* *} \cap\left(D_{i_{0}}-\{d\}\right)\right)^{*} \neq K^{*}$.

(3) $d$ is an absolutely unnecessary decision attribute iff for all $B \in \Phi_{C}$, satisfying $\left(B^{* *}-\{d\}\right)^{*}=$ $B^{*}$ and for all $D_{i}$, with $i \in \Upsilon$, satisfying $\left(B^{* *} \cap\left(D_{i}-\right.\right.$ $\{d\}))^{*}=B^{*}$

Proof. (1) $d \in C$ is an absolutely necessary decision attribute of $S$ if and only if $C-\{d\}$ is not consistent set. It is equivalent to the fact that there exists $B \in \Phi_{C}$ satisfying $\left(B^{* *}-\{d\}\right)^{*} \neq B^{*}$ by Theorem 6 .

(2) $d \in C$ is a relatively necessary decision attribute of $S$ if and only if $d$ is not a core attribute and there exists a reduct $D_{i_{0}}$, with $i_{0} \in \Upsilon$, satisfying $d \in D_{i_{0}}$. Therefore the conclusion can be obtained from (1) and Theorem 6.

(3) It follows immediately from (1) and (2).

Example 2. In the decision formal context $S=$ $(U, A, I, C, J)$ shown in Table 1, by Theorem 7, we can verify that $\{a, b, e\}$ are the absolutely necessary condition attributes set and $\{c, d, f\}$ are the absolutely unnecessary condition attributes set; $\{g, k, l\}$ are the absolutely necessary decision attributes set, and $\{h, m, n\}$ are the absolutely unnecessary decision attributes set.

\section{Conclusions}

Attribute reduction in the decision formal contex$t$ is one of the important problems in knowledge discovery. In this paper, we propose the condition reduct and the decision reduct based on maximal rules. The reducts preserve all the condition extensions and decision extensions related to the original maximal rules. After eliminating the superfluous attributes, the depiction of the maximal rule becomes 
more concise and accurate. It is proven that the maximal rules in the reduced decision formal context can be obtained based on the original maximal rules, the condition consistent set and the decision consistent set. The related discernibility matrix is constructed to compute all the reducts via Boolean approach. Based on the maximal rules, all the attributes are classified into three types. The characteristics of these types of attributes are also analyzed. Our further work is to study attribute reduction method based maximal rules in the fuzzy decision formal concept lattices and explore its mathematical property.

\section{Acknowledgments}

This work is supported by National Natural Science Foundation of China under Grant 61170107, 61350004 and 61073125, National Science Fund for Distinguished Young Scholars under Grant 50925625, the Research Fund for the Doctoral Program of Higher Education of China (No. 20101303110004) and the Fundamental Research Funds for the Central Universities (No. HIT. NSRIF. 2013091).

\section{References}

1. R. Agrawal, T. Imielinski and A. Swami, "Mining association rules between sets of items in large databases," Proceedings of the ACM SIGMOD Conference on Management of data, Washington, DC, USA: ACM Press, 207-216 (1993). doi:10.1145/ 170035. 170072

2. P. Y. Bastide, R. Taouil and L. Lakhal, "Discovering frequent closed item sets for association rules," Proceedings of 7th International Conference Database Theory (ICDT99), Jerusalem, Israel: Springer-Verlag, 398-416 (1999). doi:10.1007/ 3-540-49257-7_25

3. R. Belohlavek, B. D. Baets, J. Outrata and V. Vychodil, "Inducing decision trees via concept lattices," International Journal of General Systems, 38, 15635104 (2009). doi : 10 . 1080/03081070902857563

4. A. Berry and A. Sigayret, "Representing a concept lattice by a graph," Discrete Applied Mathematics, 144, 27-42 (2004). doi : 10.1016/j.dam.2004.02.016
5. C. Carpineto and G. Romano, "A lattice conceptual clustering system and its application to browsing retrieval," Maching Learning, 10, 95-122 (1996). doi : 10.1007/BF00058654

6. R. Cole, P. Eklund and G. Stumme, "Document retrieval for e-mail search and discovery using formal concept analysis," Applied Artificial Intelligence, 17, 257-280 (2003). doi : 10.1080/713827120

7. B. Ganter and R. Wille, "Formal concept analysis: Mathematical Foundations," Springer, Berlin, 1999.

8. L. K. Guo, F. P. Huang, Q. G. Li and G. Q. Zhang, "Power contexts and their concept lattices," Discrete Mathematics, 311, 2049-2063 (2011). doi : 10 . $1016 / j$.disc. 2011.04 .033

9. S. K. Harms and J. S. Deogun, "Sequencial accociation rule with time lags," Journal of Intelligent Information Systems, 22, 7-22 (2004). doi:10.1023/A : 1025824629047

10. Y. Leung and D. Y. Li, "Maximal consistent block technique for rule acquisition in incomplete information systems," Information Sciences, 153, 85-106 (2003). doi : 10.1016/S0020-0255 (03)00061-6

11. J. H. Li, C. L. Mei and Y. J. Lv, "A heuristic knowledge-reduction method for decision formal contexts," Computers and Mathematics with Applications, 61, 1096-1106 (2011). doi:10.1016/j.camwa. 2010.12 .060

12. J. H. Li, C. L. Mei and Y. J. Lv, "Knowledge reduction in formal decision contexts based on an orderpreserving mapping," International Journal of General Systems, 41, 143-161 (2012). doi:10.1080/ 03081079.2011 .634410 .

13. M. Z. Li and J. S. Mi, "The strong direct product of formal contexts," Information Sciences, 226, 47-67 (2013). doi:10.1016/j.ins.2012.10.032

14. M. Liu, M. W. Shao, W. X. Zhang and C. Wu, "Reduction method for concept lattices based on rough set theory and its application," Computers and Mathematics with Applications, 53, 1390-1410 (2007). doi: 10.1016/j. camwa. 2006.03 .040

15. E. Marczewski, "A general scheme of independence in mathematics," Bulletin de L Academie of Polonaise des Sciences-Serie des Sciences Mathematiques Astronomiques et Physiques, 6, 731-736 (1958).

16. J. Medina, "Relating attribute reduction in formal, object-oriented and property-oriented concept lattices," Computers and Mathematics with Applications 64, 1992-2002 (2012). doi:10.1016/j.camwa. 2012.03 .087

17. J. Medina, M. Ojeda-Aciego and J. Ruiz-Calviño, "Relating generalized concept lattices and concept lattices for non-commutative conjunctors," Applied Mathematics Letters, 21, 1296-1300 (2008). doi : 10 . 1016/j.aml.2007.12.026

18. J. Medina, M. Ojeda-Aciego and J. Ruiz-Calvi, "For- 
mal concept analysis via multi-adjoint concept lattices," Fuzzy Sets and Systems, 160, 130-144 (2009). doi:10.1016/j.fss.2008.05.004

19. J. S. Mi, Y. Leung and W. Z. Wu, "Approaches to attribute reduction in concept lattices induced by axialities," Knowledge-Based Systems, 23, 504-511 (2010). doi : 10.1016/j.knosys.2010.03.007

20. T. T. Nguyen, S. C. Hui and K. Y. Chang, "A latticebased approach for mathematical search using Formal Concept Analysis," Expert Systems with Application$s, 39,5820-5828$ (2012). doi:10.1016/j.eswa. 2011.11 .085

21. T. Oyama, K. Kitano and T. K. Satou, "Extraction of knowledge on protein-protein interaction by association rule discovery," Bioinformatics, 18, 705-714 (2002). doi:10.1093/bioinformatics/18.5.705

22. Z. Pawlak, "Rough sets," International Journal of Computer and Information Science, 11, 341-356 (1982). doi:10.1007/BF01001956

23. J. R. Quinlan, "Simplifying decision trees," International Journal of Man-Machine Studies, 27, 221-234 (1987). doi:10.1016/S0020-7373(87)80053-6

24. K. S. Qu, Y. H. Zhai, J. Y. Liang and M. Chen, "Study of decision implications based on formal concept analysis," International Journal of General Systems, 36, 147-156 (2007). doi:10.1080/ 03081070600913650

25. Y. C. Shao and K. Y. Qin, "Fuzzy Soft Sets and Fuzzy Soft Lattices," International Journal of Computational Intelligence Systems, 5, 1135-1147 (2012). doi: $10.1080 / 18756891.2012 .747667$

26. P. Tonella, "Using a concept lattice of decomposition slices for program understanding and impact analysis," IEEE Transactions on Software Engineering, 29, 495-509 (2003). doi :10.1109/TSE. 2003. 1205178

27. X. Wang and W. X. Zhang, "Relations of attribute reduction between object and property oriented concept lattices," Knowledge Based Systems, 21, 398-403 (2008). doi:10.1016/j.knosys.2008.02.005

28. H. Wang and W. X. Zhang, "Approaches to knowl- edge reduction in generalized consistent decision formal context," Mathematical and Computer Modelling, 48, 1677-1684 (2008). doi : 10.1016/j.mcm. 2008. 06.007

29. L. Wei, J. J. Qi and W. X. Zhang, "Attribute reduction theory of concept lattice based on decision formal contexts," Science in China Series F: Information Sciences, 51, 910-923 (2008). doi:10.1007/ s11432-008-0067-4

30. R. Wille, "Restructuring lattice theory: an approach based on hierarchies of concepts," Formal Concept Analysis, 314-339 (2009). doi:10.1007/ 978-3-642-01815-2_23

31. K. E. Wolff, "A conceptual view of knowledge bases in rough set theory," Lecture Notes in Computer Science, 220-228 (2005). doi:10.1007/ 3-540-45554-X_26

32. W. Z. Wu, Y. Leung and J. S. Mi, "Granular computing and knowledge reduction in formal context," IEEE Transactions on Knowledge and Data Engineering, 21, 1461-1474 (2009). doi:10.1109/TKDE. 2008. 223

33. Y. Y. Yao, "A comparative study of formal concep$\mathrm{t}$ analysis and rough set theory in data analysis," In: S. Tsumoto, R. Slowinski, H. Jan Komorowski, J.W. Grzymala-Busse (Eds.) Springer, Berlin, 59-68 (2004). doi : 10.1007/978-3-540-25929-9_6

34. H. Z. Yang, Y. Leung, and M. W. Shao, "Rule acquisition and attribute reduction in real decision formal contexts," Soft Computing, 15, 1115-1128 (2011). doi:10.1007/s00500-010-0578-y

35. L. Yang, Y. H. Wang and Y. Xu, "A Combination Algorithm of Multiple Lattice-Valued Concept Lattices," International Journal of Computational Intelligence Systems, 6, 881-892 (2013). doi:10.1080/ 18756891.2013 .807109

36. W. X. Zhang, L. Wei and J. J. Qi, "Attribute reduction in concept lattice based on discernibility matrix," Lecture Notes in Computer Science, 3642, 157-165 (2005). doi : 10.1007/11548706_17 